\title{
Quelques propositions au sujet des fondations profondes
}

\section{A. MONNET}

V. BERNHARDT

Géoconseil

10, avenue Newton 92350 Le Plessis-Robinson
Dans une première partie, I'analyse d'un essai de chargement axial de pieu permet de rappeler les données expérimentales fondamentales.

La seconde partie propose un modèle de comportement des fondations profondes. Ce modèle est appliqué au cas de l'essai et les résultats de calcul sont confrontếs aux mesures.

La conclusion met en question l'interprétation habituelle des essais de chargement à la lueur des apports du caicul en déplacement.

\section{Some suggestions about deep foundations}

In the second part, a very simple model for the loading of deep foundations is proposed and is used to make a simulation of the pile test. The model uses only two parameters for each soil layer. However the model is able to describe with accuracy the axial loading of a deep foundation.

In the conclusion, the prevalent interpretation of the pile load tests is discussed, subsequently to the results of the computation. 


\section{Essai de chargement}

L'élément de fondation est un cas type de fondation profonde, un pieu foré de diamètre nominal $0,60 \mathrm{~m}$ avec une virole de $0,80 \mathrm{~m}$ entre les cotes $-0,30$ et $+3,40$ (cet élargissement n'est pas représenté sur le croquis).

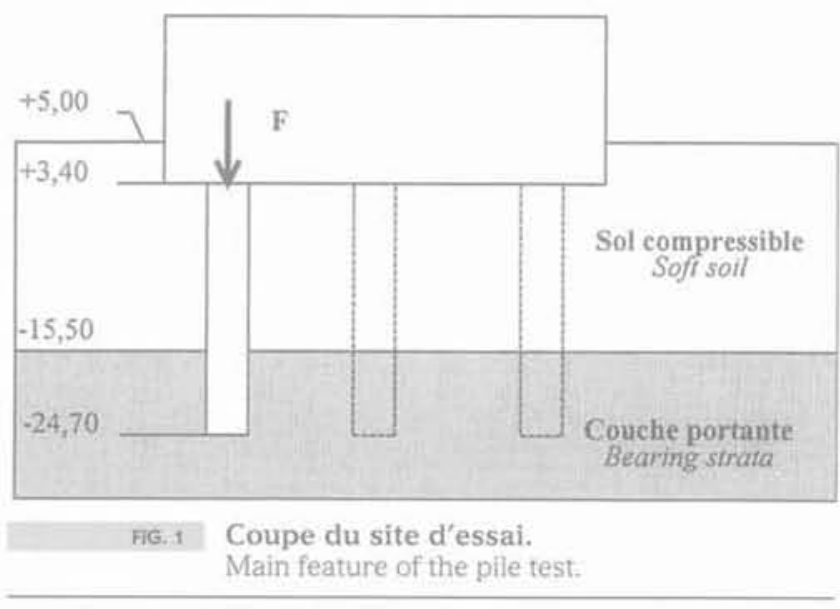

Les données utilisées proviennent d'un rapport d'essai préliminaire à la réalisation d'un chantier. Il s'agit d'un essai de routine, non pas d'un essai à but de recherche. L'interprétation initiale se limite à la détermination de la capacité portante, sans chercher à assurer la cohérence des mesures. Comme il s'agit d'un document d'archives, il n'est plus possible d'obtenir les informations complémentaires qui permettraient d'assurer cette cohérence.

C'est la situation à laquelle le concepteur se trouve en général confronté. C'est pourquoi cet ensemble de données a été retenu, sans rechercher un essai où toutes les incertitudes auraient été résolues.

Toutes les données brutes jugées significatives sont citées, ce qui permet éventuellement une autre interprétation que celle qui est proposée ici.

\section{1}

\section{Description de l'essai}

\section{4 \\ Géotechnique du site}

Le site est reconnu par plusieurs forages pressiométriques. La figure 2 superpose la coupe à l'emplacement du pieu d'essai et les résultats pressiométriques sur un sondage proche.

La couche portante, au-dessous de - 15,50, est constituée d'une couche de sable graveleux assez compacte, avec des pressions limites (pl) croissant de 1,5 à 2,5 Mpa, puis du gneiss, altéré sur 3,0 mètres, avec des pl de 2,5 Mpa, et compact ensuite, avec des pl croissant rapidement. Selon le rapport de forage, la base du pieu est au contact de la transition gneiss altéré/gneiss compact.

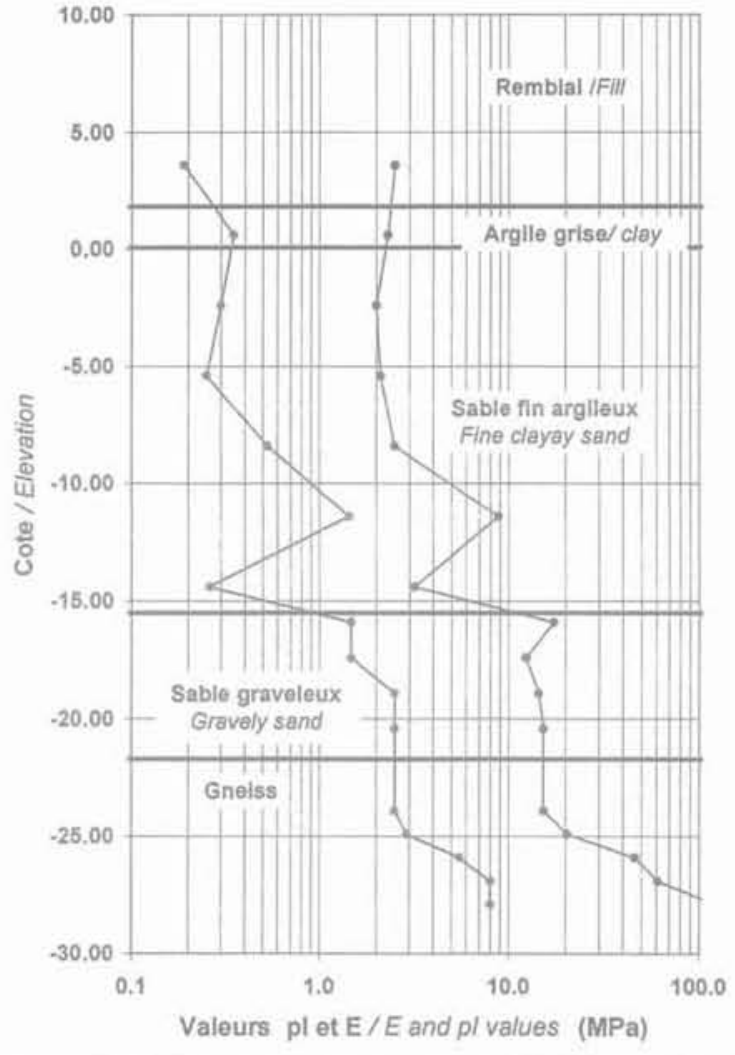

fiG. 8 Coupe sur le pieu et profil pressiométrique à proximité.

Test pile soil profile and pressuremeter results in the vicinity.

\section{4his}

\section{Instrumentation}

L'instrumentation comporte une cellule manométrique pour mesurer la charge appliquée en tête et des comparateurs sur base fixe pour la mesure du tassement de la tête du pieu.

Un extensomètre multiple est installé avec des bloqueurs aux cotes précisées par le tableau I pour mesurer les raccourcissements du pieu à chaque palier de chargement. Le principe de fonctionnement de l'extensomètre consiste à mesurer à l'aide d'une jauge la déformation moyenne entre deux bloqueurs.

TABLEAUI Extensométrie.

Extensometer elevation.

\begin{tabular}{l|c|c|c|}
\hline Repere & $\begin{array}{c}\text { Cote } \\
\text { du bloqueur } \\
\text { Blocking device } \\
\text { elevation }\end{array}$ & $\begin{array}{c}\text { Cote moyenne } \\
\text { de mesure } \\
\text { Mean measure } \\
\text { elevation }\end{array}$ & $\begin{array}{c}\text { Longueur } \\
\text { d'application } \\
\text { Extension } \\
\text { of the measure }\end{array}$ \\
\hline 1 & 2,90 & & \\
2 & 1,90 & 2,40 & 1,00 \\
3 & $-2,10$ & $-0,10$ & 4,00 \\
4 & $-7,10$ & $-4,60$ & 5,00 \\
5 & $-12,10$ & $-9,60$ & 5,00 \\
6 & $-17,10$ & $-14,60$ & 5,00 \\
7 & $-19,10$ & $-18,10$ & 2,00 \\
8 & $-21,10$ & $-20,10$ & 2,00 \\
9 & $-22,10$ & $-21,60$ & 1,00 \\
10 & $-23,10$ & $-22,60$ & 1,00 \\
11 & $-24,10$ & $-23,60$ & 1,00 \\
\hline
\end{tabular}


TAELEAU II Paliers de chargement et mesures extensométriques $\left(x 10^{-6}\right)$. Loading steps and extensometer readings $\left(\times 10^{-6}\right)$.

\begin{tabular}{|c|c|c|c|c|c|c|c|c|c|c|c|c|}
\hline \multirow[b]{2}{*}{ Cote } & \multicolumn{12}{|c|}{ Pas de chargementiL.oading steps (MN) } \\
\hline & 0.25 & 0,50 & 0.75 & 1,00 & 1.25 & 1,50 & 1,75 & 2,00 & 2,25 & 2,50 & 2,75 & 3,00 \\
\hline 2,40 & 19,5 & 38 & 56 & 74 & 95 & 114 & 136 & 153 & 173 & 194 & 215 & 236 \\
\hline$-0,10$ & 18 & 40 & 60 & 82 & 104 & 124 & 148 & 168 & 190 & 212 & 234 & 260 \\
\hline$-4,60$ & 16 & 36 & 54 & 72 & 93 & 114 & 138 & 161 & 185 & 208 & 233 & 258 \\
\hline$-9,60$ & 13 & 30 & 47 & 67 & 89 & 108 & 133 & 156 & 177 & 200 & 222 & 243 \\
\hline$-14,60$ & 10 & 21 & 33 & 47 & 62 & 77 & 92 & 108 & 124 & 139 & 155 & 170 \\
\hline$-18,10$ & 7 & 18 & 30 & 42 & 56 & 70 & 86 & 101 & 114 & 128 & 140 & 152 \\
\hline$-20,10$ & 4 & 8 & 12 & 18 & 23 & 28 & 34 & 40 & 45 & 53 & 60 & 65 \\
\hline$-21,60$ & 2 & 6 & 9 & 12 & 15 & 20 & 25 & 30 & 34 & 39 & 44 & 48 \\
\hline$-22,60$ & 1 & 3 & 5 & 7 & 8 & 11 & 13 & 17 & 20 & 23 & 26 & 30 \\
\hline$-23,60$ & 0,5 & 1 & 3 & 5 & 7 & 9 & 11 & 13 & 15 & 19 & 22 & 25 \\
\hline
\end{tabular}

\section{tili.3.3.}

\section{Programme de chargement}

La charge a été appliquée par paliers croissants. Le tableau II donne la valeur des paliers de chargement.

\section{2}

\section{Mesures}

\section{1}

\section{Volume de béton}

La mesure des volumes de béton mis en place montre que le pieu présente en réalité un diamètre moyen de $0,68 \mathrm{~m}$ dans la partie basse et de $0,80 \mathrm{~m}$ dans la partie haute.

Le faible nombre de mesures ne permet pas de préciser de manière plus fine la distribution des diamètres au-dessous de la base de la virole de tête.

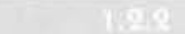

\section{Mesures des extensomètres}

Le tableau II donne le relevé des mesures brutes sur les extensomètres (les valeurs sont données en microns/mètre).

\section{3}

\section{Tassement en tête}

La mesure du tassement en tête (tableau III et figure 3) montre que le déplacement n'est ni linéaire, ni réversible.

Ce type de comportement est tout à fait typique, et, en ce sens, cet essai est représentatif du comportement général d'une fondation profonde sous charge.

\section{4}

\section{Cohérence des mesures}

Le tassement mesuré en tête devrait être cohérent avec les mesures sur les extensomètres. Or, la prise en
TABLEAUIIII Tassement de la tête. Pile head settlement

\begin{tabular}{l|c|c|}
$\begin{array}{l}\text { Charge en tète } \\
\text { Loading step }\end{array}$ & \multicolumn{2}{|c}{$\begin{array}{c}\text { Tassement } \\
\text { Settlement (mm) }\end{array}$} \\
\hline (MN) & $\begin{array}{c}\text { Au chargement } \\
\text { Loading }\end{array}$ & $\begin{array}{c}\text { Déchargement } \\
\text { Enloading }\end{array}$ \\
\hline 0,00 & 0,00 & 1,61 \\
0,25 & 0,20 & 2,10 \\
0,50 & 0,50 & 2,50 \\
0,75 & 0,80 & 2,90 \\
1,00 & 1,10 & 3,15 \\
1,25 & 1,50 & 3,50 \\
1,50 & 1,90 & 3,85 \\
1,75 & 2,35 & 4,20 \\
2,00 & 2,80 & 4,50 \\
2,25 & 3,30 & 4,75 \\
2.50 & 3,80 & 4,90 \\
2,75 & 4,40 & 5,00 \\
3,00 & 5,11 & 5,11 \\
\hline
\end{tabular}

Charge en tête / Test load (MN)

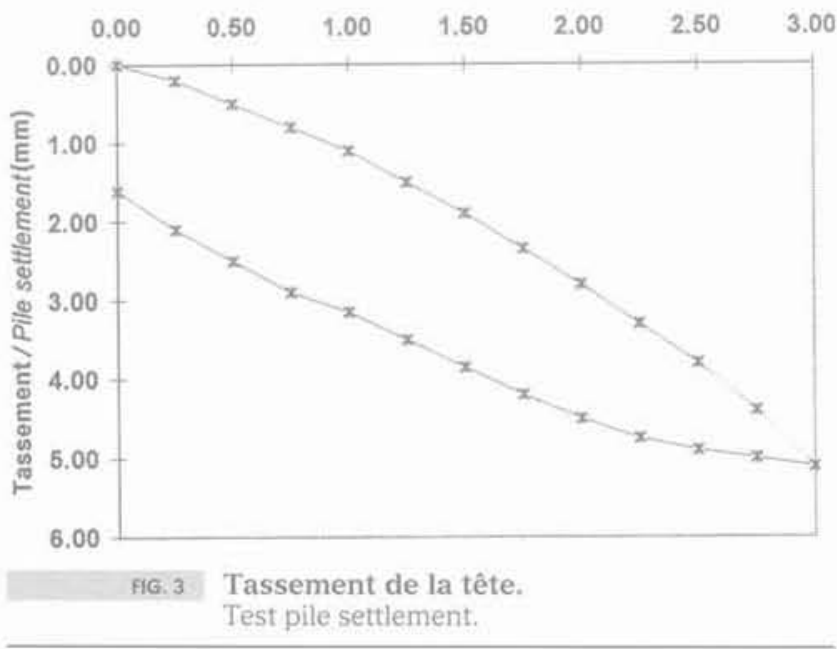

compte des valeurs brutes des mesures sur les extensomètres fait apparaitre un soulèvement de la pointe en début de chargement, ce qui est inadmissible. 
Une première interprétation pourrait être que la base $\alpha$ fixe $»$ de mesure des tassements ne l'est pas autant qu'elle le devrait. Mais le rapport d'essai précise que les mesures de tassements de la tête sur comparateur sont recoupées par des mesures topographiques sur base éloignée.

Reste l'hypothèse d'un mauvais calibrage des mesures sur les extensomètres. Il faut appliquer un facteur de 0,69 aux valeurs brutes pour que les raccourcissements soient compatibles avec un tassement réaliste de la pointe.

Avec ces valeurs corrigées, et les diamètres réels estimés du pieu, il est possible de calculer le module du béton ( $35000 \mathrm{MPa}$ ) qui met en accord la charge calculée sur le premier tronçon de mesure avec la charge mesurée en tête.

Ceci fait, il est possible de calculer la distribution des charges sur la longueur du pieu pour chaque palier de chargement (Fig. 4).

Une analyse qualitative de ces courbes montre que la charge se dissipe très peu entre les cotes $-4,60$ et $-9,60$ comme entre les cotes - 14,60 et - 18,10 ce qui correspond aux zones indiquées comme lâches par la reconnaissance pressiométrique.

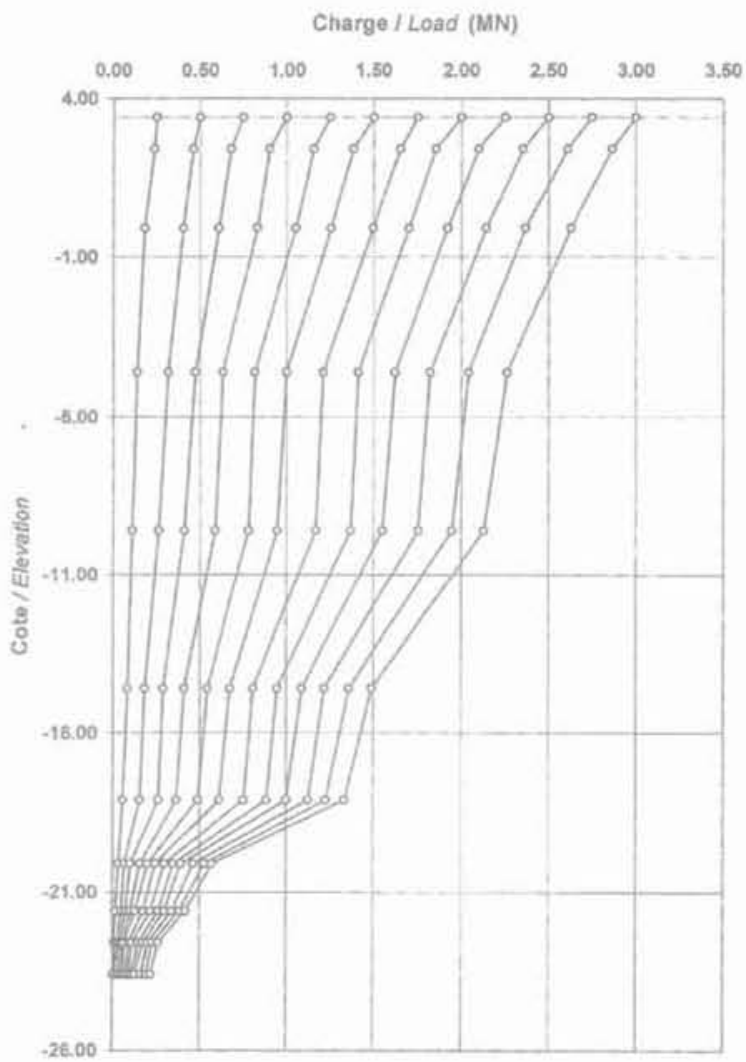

FIG. 4 Distribution des charges.

Axial load distribution with elevation.

\section{3}

\section{Interprétation}

\section{I.3il}

\section{Résistance de pointe}

A partir de la charge et du tassement calculés au niveau du dernier bloqueur, il est possible de reconstituer la mobilisation de la résistance de pointe (Fig. 5).
La dernière mesure correspond à une charge en pointe de $219 \mathrm{kN}$. Il est facile d'inscrire avec une corrélation presque parfaite une courbe du second degré dans le nuage de points, comme le propose la figure 5 .

Il faut remarquer qu'il serait également possible de distinguer trois comportements successifs en fonction de la charge :

- un comportement linéaire aux très faibles charges, inférieures à $0,05 \mathrm{MN}$;

- un premier comportement d'écrouissage jusqu'à une charge de l'ordre de 0,13 MN ;

- puis un second au-delà, sur une base différente.

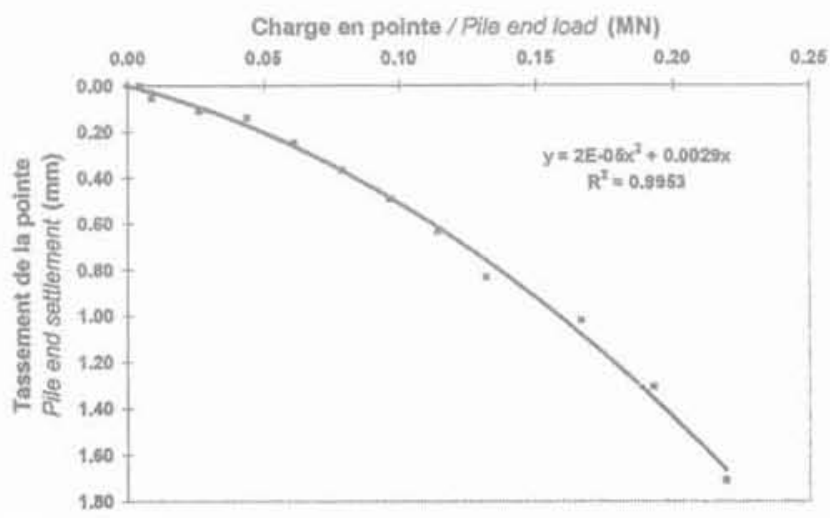

56.5 Mobilisation de la résistance de pointe. End bearing load building-up.

\section{(13) \\ Frottement latéral}

En rapportant la variation de charge entre deux niveaux de bloqueurs au tassement moyen des deux bloqueurs, il est possible de tracer la courbe de mobilisation du frottement latéral unitaire $q_{\text {. }}$.

\section{Sols compressibles}

La figure 6 regroupe les valeurs du frottement latéral sur les tronçons du pieu situés dans les sols compressibles. Comme pour les couches suivantes, la dispersion des résultats d'un niveau de mesure à l'autre est très importante, alors que le comportement à un niveau donné est relativement régulier.

La variabilité des caractéristiques du sol peut être à l'origine de cette dispersion. Et en effet, le niveau - 4,60 qui donne les plus mauvais résultats correspond au "creux ») de la courbe pressiométrique. Mais ces variations peuvent également provenir de variations dans le diamètre local du pieu, puisque les valeurs sont calculées sur la base d'un diamètre moyen.

Si on admet de plus qu'il est possible que le diamètre réel soit plus important dans les zones où le sol présente les caractéristiques les plus faibles, seule la moyenne des comportements sur la hauteur de la couche serait quantitativement significative.

La moyenne sur les trois niveaux (courbe épaisse) montre que le cisaillement atteint un palier, avec une valeur proche de $32 \mathrm{kPa}$ pour un déplacement de $3,5 \mathrm{~mm}$ en fin d'essai. 


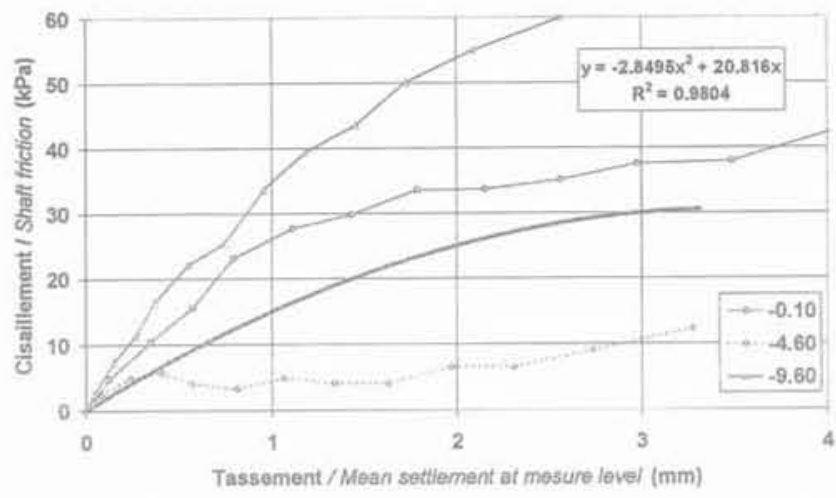

Fic. Mobilisation du frottement latéral dans les sols compressibles.

Shaft friction bullding up in the soft soils.

\section{Sables graveleux}

La mobilisation du frottement latéral dans les sables graveleux (Fig. 7) montre qu'en moyenne, en fin d'essai, un palier à $100 \mathrm{kPa}$ pourrait avoir été atteint pour un déplacement inférieur à $2 \mathrm{~mm}$.

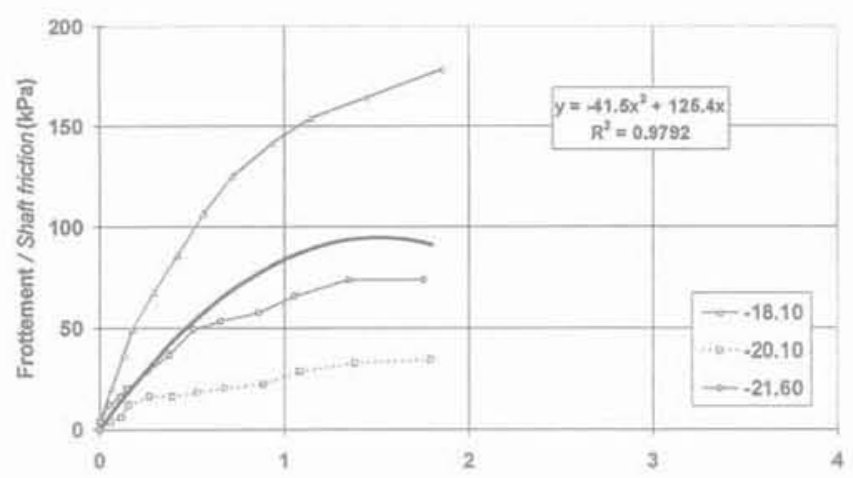

fiG.7 Mobilisation du frottement latéral dans les sables graveleux.

Shaft friction building up in the gravely sand.

La rigidité est plus importante que pour les sols compressibles. Sur les moyennes, le rapport des rigidités est de plus de 7, pour un rapport des cisaillements limites de 3 environ.

\section{Gneiss altéré}

La mobilisation du frottement latéral dans le gneiss altéré (Fig. 8) atteint en moyenne $60 \mathrm{kPa}$ en fin d'essai, pour un déplacement très proche de celui mesuré dans les sables graveleux. Il semble qu'ici le cisaillement limite ne soit pas encore atteint, mais proche.

Sur les moyennes, le rapport des rigidités comme le rapport des cisaillements limites entre le sable graveleux et le gneiss altéré sont voisins de 1,6.

\subsection{3.}

\section{Synthèse}

La figure 4 montre que la charge appliquée en tête de la fondation est reprise partiellement par une réaction de cisaillement le long du fût, le frottement latéral $F_{S}$. Le solde des efforts est repris par une réaction sous la base de la fondation, la réaction de pointe, $F_{B}$.

$$
F=F_{S}+F_{B}
$$

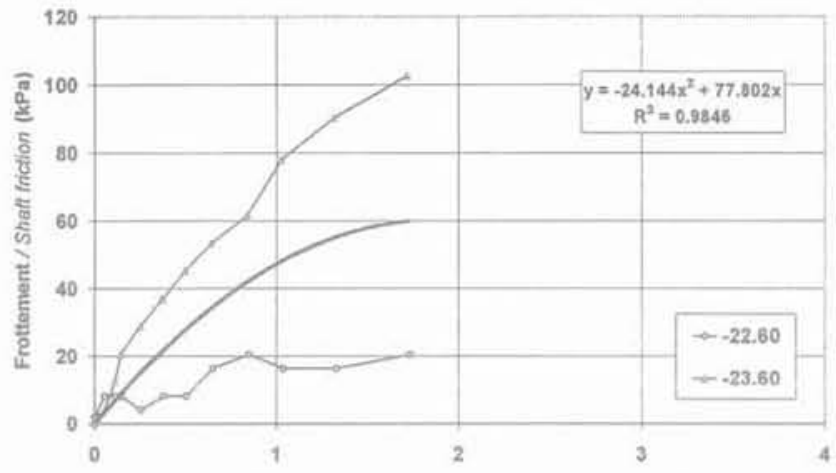

FIG. 8 Mobilisation du frottement latéral dans le gneiss altéré,

Shaft friction building up in the weathered gneiss.

Bien que l'allure de la distribution des efforts le long du pieu soit similaire pour les pas successifs de chargement, une analyse plus fine montre que la part de la résistance de pointe augmente avec la charge (elle passe de un peu moins de $2 \%$ de la charge totale au premier pas de chargement à près de $10 \%$ à la fin de l'essai). En termes familiers, le frottement latéral est progressivement saturé avec l'augmentation de la charge.

Le frottement latéral unitaire, $q_{S}$, croit initialement de manière parabolique avec le tassement et est borné par une valeur limite, le frottement ultime, $q_{s u}$ qui est fonction de la compacité, mais aussi de la nature du sol. A titre d'exemple, le gneiss altéré, qui n'est pas discernable du sable graveleux dans les résultats de l'essai pressiométrique, présente des caractéristiques nettement inférieures.

La contrainte en pointe, $q_{B}$, croît également de manière sensiblement parabolique avec le tassement. Dans le cas particulier, l'existence d'une contrainte limite en pointe, $q_{B y}$, n'est pas mise en évidence. Mais d'autres expériences (les essais pénétrométriques, le fonçage et le battage des pieux) attestent de sa réalité.

\section{4}

\section{Conclusion}

Cet essai a été retenu au hasard, pour la seule raison que les séries de données étaient complètes et d'origines variées. L'objectif était double :

- d'une part, de disposer d'une base pour tester les propositions de la partie suivante

- et, d'autre part, de rappeler la nature expérimentale des notions de base qui sont utilisées.

Des interprétations ont été nécessaires pour rendre cohérentes les diverses informations. Finalement cet essai de routine permet de retrouver la plupart des notions classiques.

Mais il est clair que ces notions portent sur des moyennes. Le cas du frottement latéral dans le gneiss altéré (Fig. 8) est significatif. L'évolution de la mobilisation du cisaillement à chaque niveau semble aléatoire. Cependant, à un glissement sur le niveau - 22,60 correspond un rebond sur le niveau $-23,60$ si bien que la régularité de la mobilisation se retrouve sur la moyenne des deux mesures. Il est probable que les irrégularités relevées figure 5 pour la mobilisation des efforts en pointe ont la même origine. 
L'objet de la section qui suit est de proposer un modèle analytique pour le comportement au chargement d'une fondation profonde. Les remarques qui précèdent posent les limites d'un modèle de ce type : il est possible d'en attendre au mieux une simulation générale du comportement, sans possibilité de reproduire le détail à chaque niveau.

\section{Remarque}

Dans le texte, $\approx$ cisaillement s) est parfois utilisé pour frottement latéral unitaire. De même « glissement $)$ peut être utilisé pour distorsion ou pour rupture en cisaillement ou encore dans son sens courant, pour alléger la lecture. Le contexte précise le sens.

\section{2}

\section{Modèle ELS}

Le modèle ELS de comportement de la fondation profonde doit permettre d'évaluer les tassements sous charge.

Le modèle prend en compte les bases suivantes : a) les déformations de la fondation restent dans le domaine élastique. A chaque niveau, la variation du tassement $w$ de la fondation est égale au raccourcissement élastique, soit au quotient de la charge $F$ par le produit EA de la section par son module d'élasticité,

$$
\varepsilon=\frac{\delta w}{\delta z}=\frac{F}{E A}
$$

Il existe donc toujours et partout une relation entre la charge et le tassement :

$$
F=E A \cdot \frac{\delta w}{\delta z}
$$

b) un tronçon élémentaire de l'élément de fondation est en équilibre sous l'effet de la variation de la charge $\delta F / \delta z$ et du frottement latéral $q_{s}$ sur le périmètre $p$,

$$
\frac{\delta F}{\delta z}-p \cdot q_{s}=0
$$

Il existe donc à tous les niveaux une relation entre le frottement latéral et le tassement:

$$
q_{s}=\frac{E A}{p} \cdot \frac{\delta^{2} w}{\delta z^{2}}
$$

qui peut également être présentée sous la forme :

$$
\frac{\delta^{2} w}{\delta z^{2}}-\frac{p}{E A} \cdot q_{s}=0
$$

\section{1}

\section{Élasticité linéaire}

Bien que l'analyse du comportement réel de la fondation montre que la mobilisation des cisaillements n'est pas linéaire, l'hypothèse de l'élasticité linéaire du sol est retenue en première analyse.

\subsection{1.}

\section{Mobilisation du frottement latéral}

La mobilisation du frottement latéral est linéaire visà-vis du glissement :

$$
q_{s}=-G, \frac{\delta w}{\delta r}
$$

Une observation classique des tassements du sol autour d'un élément de fondation profonde montre que celui-ci peut être approché comme inversement proportionnel à la distance au centre de la fondation de diamètre $\mathrm{R}$. Si $w$ est le tassement du pieu :

$$
\begin{aligned}
& w(r)=w \cdot R / r \\
& \frac{\delta w(r)}{\delta r}=-\frac{w \cdot R}{r^{2}}
\end{aligned}
$$

Dans ces conditions, au contact de la fondation :

$$
\frac{\delta w}{\delta r}=-\frac{W}{R}
$$

et il apparaît une équation différentielle qui définit entièrement le tassement de l'élément de fondation:

$$
\frac{\delta^{2} w}{\delta z^{2}}-\frac{p \cdot G}{E A \cdot R} \cdot W=0
$$

Une constante auxiliaire, la longueur caractéristique, $l_{7}$, peut être introduite, qui se présente comme le produit de deux valeurs, $l_{T}=d$. $m$. La première est une longueur qui est uniquement fonction de la géométrie de la fondation :

$$
d=\sqrt{\frac{R \cdot A}{p}}
$$

La seconde est une valeur sans dimension qui mesure le rapport de rigidité de la fondation à celle du sol :

$$
m=\sqrt{E / G}
$$

Avec la forme:

$$
\frac{\delta^{2} W}{\delta z^{2}}-\frac{1}{l_{T}^{2}}, W=0
$$

l'expression générale du tassement serait alors, dans un sol homogène avec élasticité linéaire, sans valeur limite du taux de frottement unitaire fondation/sol :

$$
W=B \cdot e^{2 / t}+C \cdot e^{-z / t}
$$

la compression dans l'élément de fondation:

$$
F=\frac{E A}{I_{T}} \cdot\left(B \cdot e^{z / t}-C \cdot e^{-z / t}\right)
$$

et le frottement latéral unitaire :

$$
q_{s}=\frac{E A}{p \cdot I_{T}^{2}}, w=\frac{G}{R}, w
$$

\subsection{8}

\section{Réaction de pointe}

En utilisant le modèle du pieu fictif, qui prolongerait la fondation vers le bas jusqu'à l'infini, avec l'origine des axes placée à la base de la fondation, les expressions du tassement et de la charge deviennent :

$$
w=w_{B} \cdot e^{2 / I_{R B}}
$$

et

$$
w_{B}=\frac{I_{T B}}{E A_{B}} \cdot F_{B}
$$

Dans ces expressions, l'indice B signale que la valeur en cause concerne la situation sous la base de la fondation. 


\section{Résolution}

A l'aide de ces relations, la résolution de l'ensemble du problème est immédiate, mais la solution semble présenter un intérêt limité puisque le comportement est entièrement linéaire, alors que l'expérience montre qu'il n'en est rien, et ceci dès le début du chargement.

\section{2}

\section{Non-linéarité}

Cependant, les relations introduites grâce à l'élasticité linéaire vont permettre de simuler de plus près le comportement réel.

\subsection{4}

\section{Frottement latéral}

Le comportement de la fondation profonde analysé dans la première partie montre que, en réalité, pour une couche de caractéristique donnée, le frottement unitaire est limité par une valeur ultime $q_{c y}$ et se mobilise de manière parabolique.

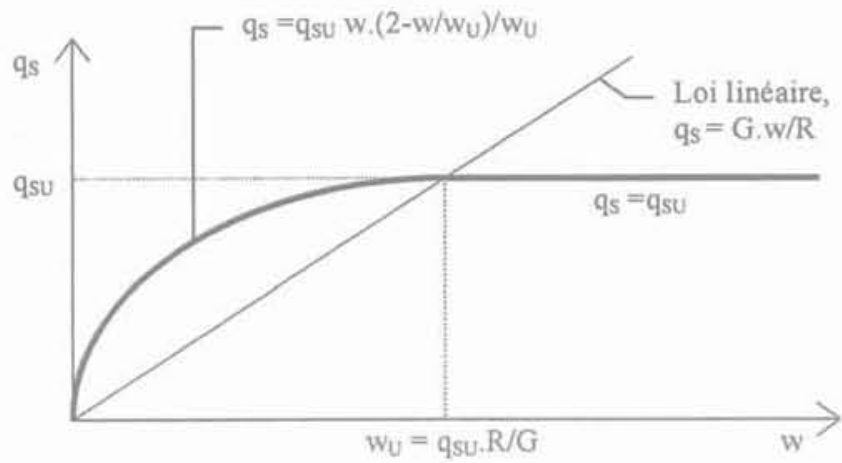

fis.9 Mobilisation du frottement latéral. Increase of shaft friction with displacement.

La loi de mobilisation du frottement latéral proposée par la figure 9 permet de prendre en compte une mobilisation parabolique du frottement latéral sans introduire d'autre paramètre supplémentaire que $q_{s u}$

\section{T20.9.}

\section{Réaction de pointe}

Pour la réaction de pointe, la situation est un peu plus complexe.

Si le sol est très rigide à la base de la fondation, il est possible d'admettre provisoirement une relation élastique linéaire entre le tassement et la charge en pointe, aussi longtemps que le tassement n'excède pas la valeur $w_{U B}$ qui mobilise le frottement limite, $q_{S U B^{\prime}}$

$$
w_{U B}=\frac{q_{S U B} \cdot p \cdot l_{T H}^{2}}{E A_{B}}
$$

Pour cette limite, la valeur de la charge en pointe est:

$$
F_{U B}=q_{S U B} \cdot p \cdot I_{T B}
$$

Au-delà de cette limite, il faut admettre un poinçonnement du sol sous la base sur une hauteur $h$, comme illustré par la figure 10.

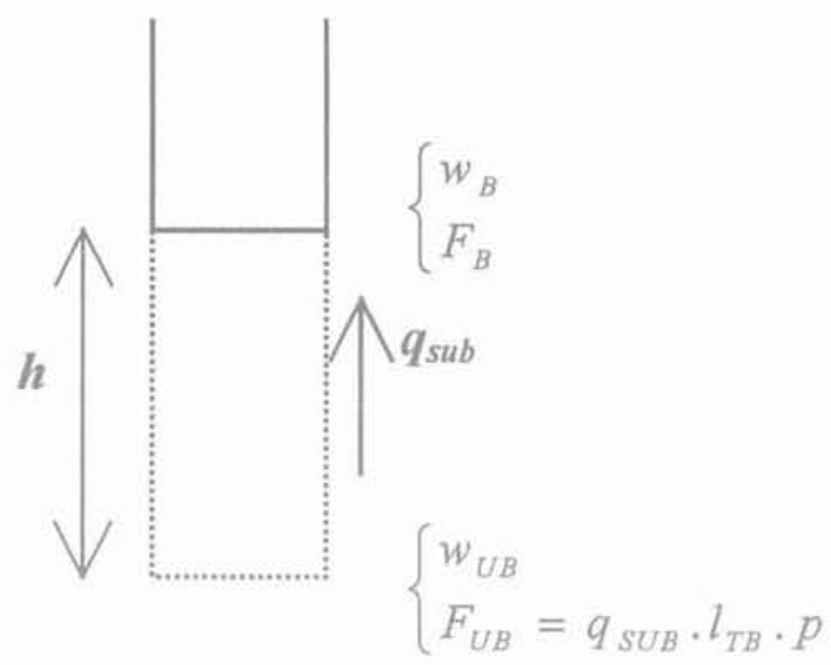

nG. 10. Modèle du pieu fíctif. The fictitious pile model.

Au-dessous, le comportement reste élastique, sans rupture.

Dans ces conditions, lorsque la charge à la base de la fondation, $F_{B}$, dépasse la valeur limite $F_{U B^{\prime}}$ le tassement de la base devient:

$$
W_{B}=W_{U B}+\frac{F_{B}^{2}-F_{U B}^{2}}{2 \cdot E A_{B} \cdot p \cdot q_{S U B}}
$$

Comme $F_{U B}^{2} /\left(E A_{B} \cdot p \cdot q_{S U B}\right)=W_{U B}$ :

$$
W_{B}=\frac{W_{U B}}{2} \cdot\left(1+\left(\frac{F_{B}}{F_{U B}}\right)^{2}\right)
$$

et inversement, $F_{B}=F_{U B} \cdot \sqrt{2 W_{B} / w_{U B}-1}$.

Au total, et en conservant 1'hypothèse d'une mobilisation linéaire du cisaillement, la loi de mobilisation de la réaction de pointe est représentée figure 11.

L'utilisation du modèle du pieu fictif prolongeant la fondation avec une limitation de la valeur du frottement latéral introduit donc un comportement non linéaire à la pointe.

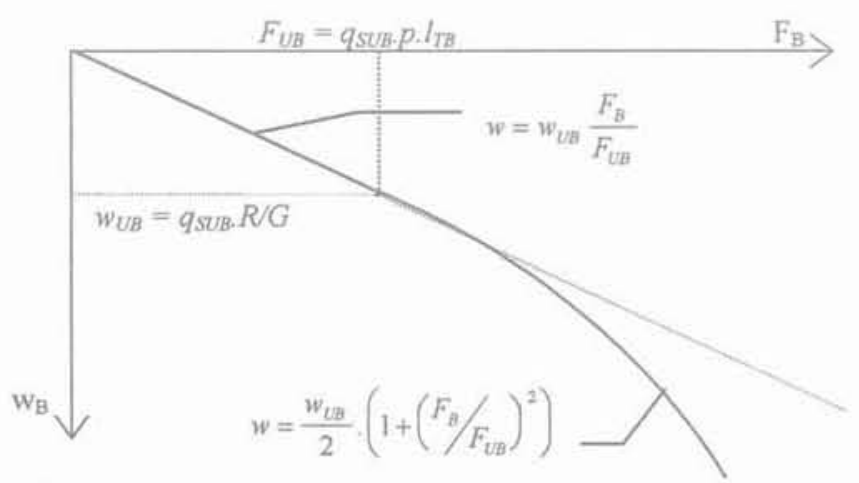

FG.11 Mobilisation de la réaction de pointe. Increase of the pile end load with the pile end settlement. 
Il est possible d'introduire l'effet de la mobilisation parabolique du frottement latéral sur le comportement de la pointe sans complication supplémentaire. Il suffit de reporter la base du calcul sur une couche très rigide (dite substratum) située plus bas que la pointe du pieu et de considérer la colonne de sol située entre la base de la fondation et le substratum comme un élément de la fondation (pieu fictif) avec le module d'Young du sol et le comportement parabolique pour la mobilisation du frottement latéral.

\section{3}

\section{Résolution}

Ces formulations ne permettent plus une résolution directe. Cependant, quelques itérations suffisent pour arriver à une solution satisfaisante.

\section{3}

\section{Valeur des paramètres}

Finalement, dans le modèle proposé, le comportement du sol est défini entièrement par deux paramètres, le frottement limite $q_{S u}$ et le module de cisaillement $G$.

\section{5}

\section{Frottement limite $q_{\text {SU }}$}

Des estimations de ce paramètre à partir des résultats d'essai in situ sont proposées par de nombreux documents. Mais dans le cas particulier oủ les résultats d'un essai sont disponibles, il est bien sûr préférable d'utiliser les valeurs moyennes mesurées à l'essai. C'est la solution retenue dans les simulations de l'essai cité en première partie qui figurent dans la suite.

A ce stade il faut noter que les documents proposent une évaluation du cisaillement limite qui est fonction du type de pieu. Sur le principe, les valeurs proposées sont valides sur la longueur du fût. Mais pour le poinçonnement sous la base, il s'agit d'une rupture sol/sol. Les cisaillements limites sont indépendants du type de pieu et ne figurent pas obligatoirement dans la même catégorie que ceux définis au-dessus de la base du pieu.

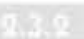

\section{Module de cisaillement sécant $G$}

Le module de cisaillement peut être estimé à partir de la mesure du module pressiométrique, $E_{p}$ :

$$
G=\frac{E p / \alpha}{2(1+v)}
$$

La valeur du coefficient de Poisson peut varier de 0 à 0,5 . La valeur maximale $(v=0,5)$ est prise en compte dans la suite.

Lorsque le module pressiométrique est mesuré sur un cycle (module alterné), il est recommandé de prendre cette valeur comme module d'Young du sol $(\alpha=1)$. Dans les autres cas, la valeur de $\alpha$ (comprise entre 1/4 et 1) en fonction de la nature du sol et du rapport Ep/pl peut être lue sur la figure 12, adaptée du fascicule 62 , titre $\mathrm{V}$, annexe $\mathrm{C} 5$.

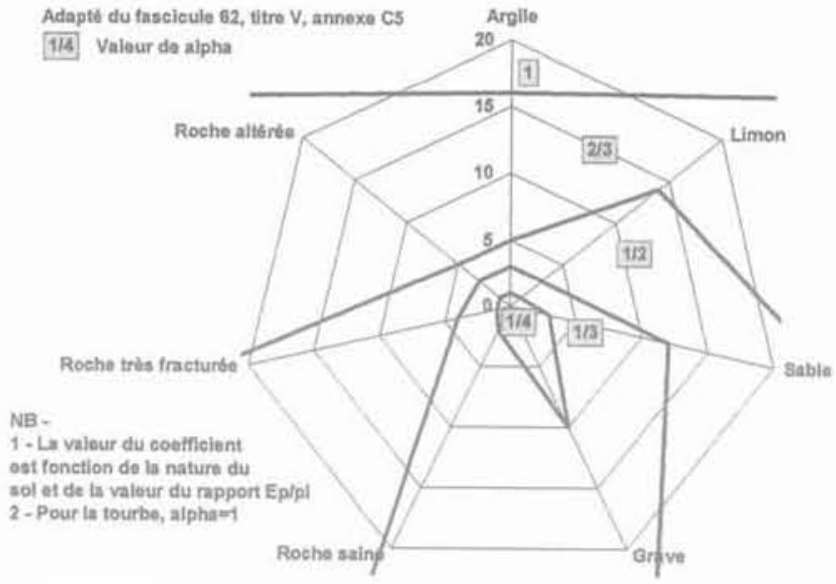

FIG, 12 Valeur du coefficient $\alpha$.

Chart of factor $\alpha$ depending on soil description and Ep/pl ratio.

\section{Application à l'essai}

\section{2-4it \\ Données}

Les données de calcul sont relevées dans le tableau IV. Les données de l'essai décrit en première partie ont été interprétées de la manière suivante : 1. la coupe de sol est celle relevée sur l'emplacement de l'essai, et non sur le sondage pressiométrique. En particulier, la couche plus résistante relevée vers -12,00 n'est pas prise en compte ;

2. les interfaces de couches sont fixées aux cotes relevées sur l'emplacement du pieu d'essai :

3. comme il est possible de le constater, les valeurs du frottement limite prévues par le DTU sont assez différentes de celles mesurées sur l'essai. Dans le cas particulier, les taux de frottement limite pour les sols lầches sont sous-estimés par le DTU, et ils sont plutôt surestimés dans les sols compacts. Pour le calcul, ce sont les valeurs mesurées à l'essai qui sont retenues quand c'est possible ;

4. les modules de sol sont estimés à partir des modules pressiométriques, avec la méthode proposée dans le chapitre qui précède ;

5. pour faire coïncider les tassements en tête et pied avec ceux mesurés sur l'essai, il a été nécessaire de supposer que $0,10 \mathrm{~m}$ de gneiss altéré subsistait sous la base du pieu.

\subsection{9}

\section{Résultats}

Le comportement de la fiche dans le modèle (courbe continue) est comparé dans la figure 13 aux résultats de l'essai (points isolés), et le comportement de la tête est représenté figure 14. Comme il a été précisé plus haut, une bonne coïncidence sur les deux ensembles n'est possible qu'en admettant que la base du pieu ne repose pas directement sur le gneiss sain. 
TAatenuiv Données de calcul.

Values used in computation.

\begin{tabular}{|c|c|c|c|c|c|c|c|c|c|c|c|c|}
\hline \multirow[b]{2}{*}{ Sol } & \multirow[t]{2}{*}{ Cote tête } & \multicolumn{2}{|c|}{ Ep (MPa) } & \multicolumn{2}{|c|}{ Pl (MPa) } & \multirow[b]{2}{*}{$\mathrm{E} / \mathrm{pl}$} & \multirow[b]{2}{*}{ alpha } & \multicolumn{2}{|c|}{ DTU 13,2 } & \multirow[b]{2}{*}{ essal } & \multicolumn{2}{|c|}{ çsu (MPa) calcul } \\
\hline & & tête & bas & tête & bas & & & classe & qsu & & tête & bas \\
\hline Remblai/fill & 3,40 & 2,50 & 2,30 & 0,19 & 0,19 & 13,2 & $1 / 3$ & Abis & 0,012 & 0,104 & 0,104 & 0,104 \\
\hline Argile/clay & 1,80 & 2,30 & 2,00 & 0,35 & 0,35 & 6,6 & $2 / 3$ & Abis & 0,020 & 0,041 & 0,041 & 0,041 \\
\hline $\begin{array}{l}\text { Sable lấche/ } \\
\text { soft sand }\end{array}$ & 0,20 & 2,00 & 2,00 & 0,25 & 0,25 & 8,0 & $1 / 3$ & Abis & 0,018 & 0,032 & 0,032 & 0,032 \\
\hline $\begin{array}{l}\text { Sable lâche/ } \\
\text { soft sand }\end{array}$ & $-5,00$ & 2,00 & 3,00 & 0,25 & 0,26 & 8,0 & $1 / 3$ & Abis & 0,015 & 0,032 & 0,032 & 0,032 \\
\hline $\begin{array}{l}\text { Sable graveleux/ } \\
\text { gravely sand }\end{array}$ & $-15,40$ & 15,00 & 15,00 & 1,46 & 2,50 & 10,3 & $1 / 3$ & B & 0,120 & 0,100 & 0,100 & 0,100 \\
\hline $\begin{array}{l}\text { Gneiss altéré/ } \\
\text { weathered gneiss }\end{array}$ & $-21,70$ & 15,00 & 15,00 & 2,50 & 2,50 & 6,0 & $2 / 3$ & C & 0,150 & 0,060 & 0,060 & 0,060 \\
\hline Gneiss & $-24,70$ & 15,00 & 40,00 & 2,90 & 2,90 & 5,2 & $2 / 3$ & C & 0,150 & & 0,060 & 0.100 \\
\hline Gneiss & $-24,80$ & 40,00 & 100,00 & 2.90 & 8,00 & 13,8 & $1 / 3$ & $\mathrm{~F}$ & $\begin{array}{l}0,200 \\
0,600\end{array}$ & & 0,100 & 0,600 \\
\hline Gneiss & $-27,90$ & 100,00 & & 8,00 & & 12,5 & $1 / 3$ & $\mathrm{~F}$ & 0,600 & & 0,600 & \\
\hline
\end{tabular}
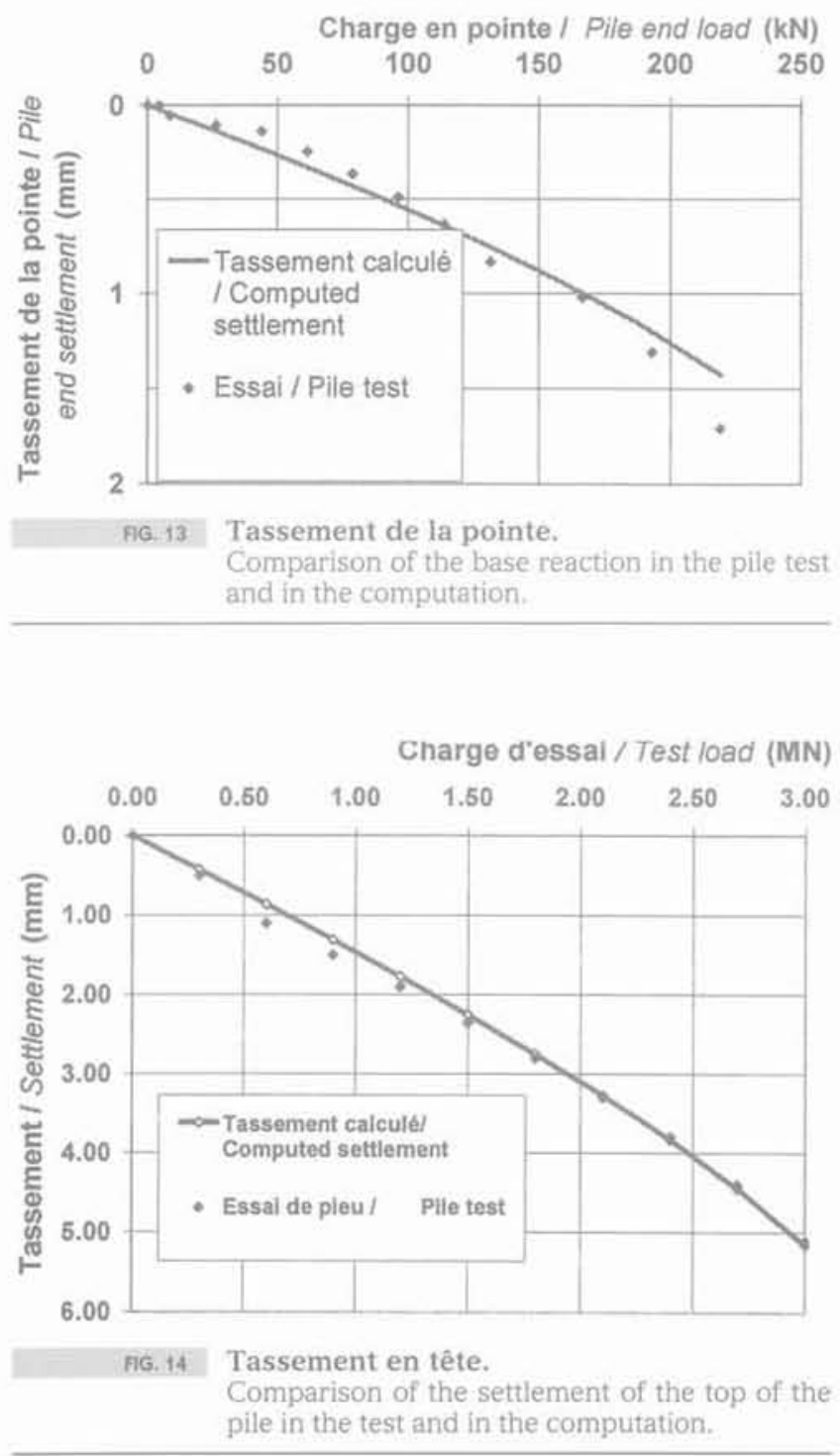

La mobilisation du frottement latéral dans les trois couches représentatives, calculée avec le modèle, est représentée figure 15 .

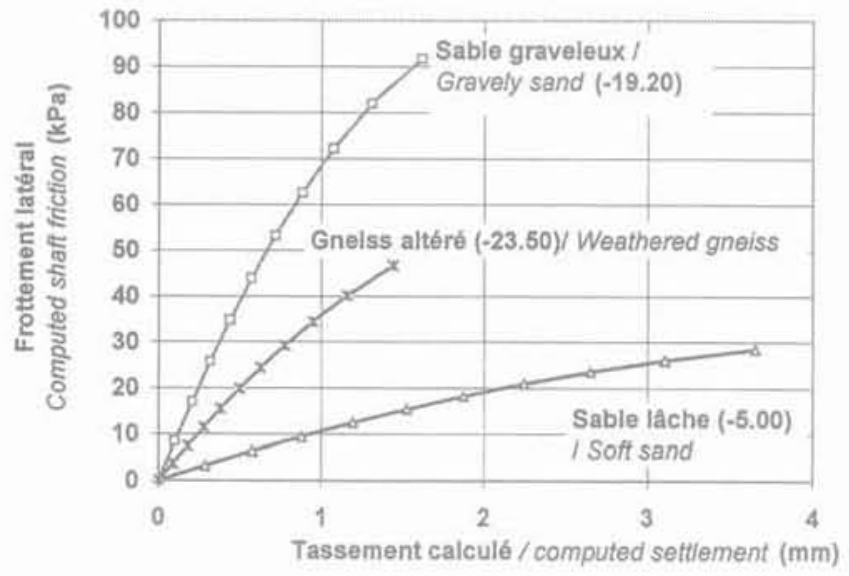

FIG.15 Mobilisation du cisaillement calculée. Increase of the shaft friction computed with the model in each soil layer.

Les cisaillements limites pris en compte sont ceux mesurés à l'essai. En revanche, la forme des courbes résulte uniquement du modèle et de l'interprétation des essais pressiométriques. La similitude entre les courbes de mobilisation du cisaillement calculées et les courbes movennes estimées à partir des résultats d'essai est remarquable.

\section{5}

\section{Discussion}

Le modèle, quî comporte uniquement deux paramètres pour décrire le comportement du sol - le frottement latéral limite, $q_{s u}$, et le module sécant de cisaillement du sol, $G_{c}$ - permet de reproduire le comportement non linéaire d'une fondation au chargement. Le modèle permet également de prévoir le poinçonnement. Par exemple, dans la figure 16, le calcul a été poussé au-delà de la charge d'essai.

Le poinçonnement qui apparaît pour une charge d'environ $3,35 \mathrm{MN}$ sur la figure 16 correspond à la saturation complète du frottement latéral, et pas à la contrainte limite en pointe. Il existe bien une plastifica- 


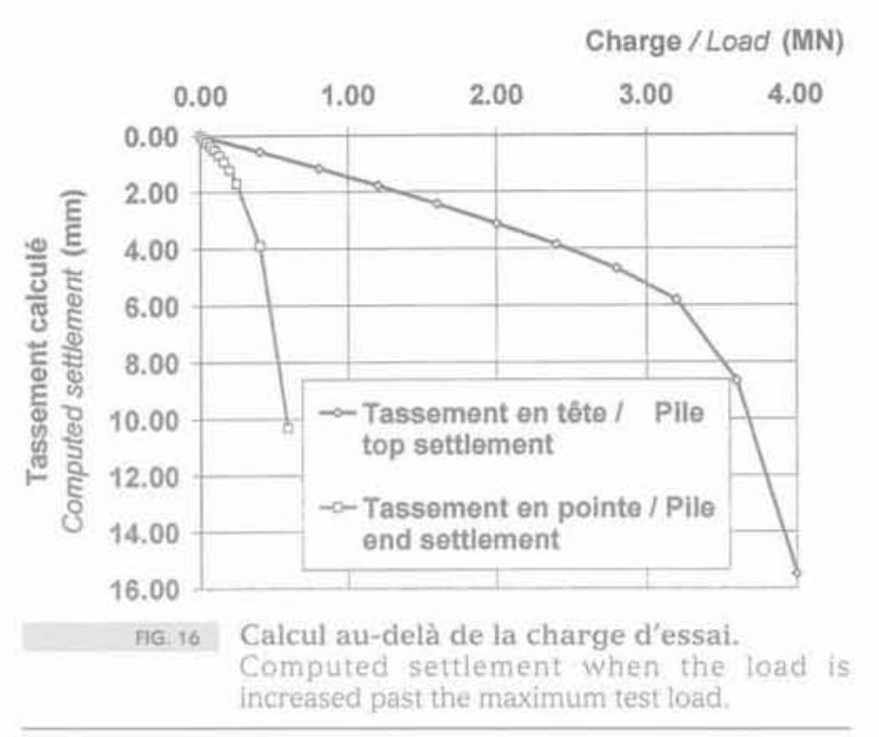

tion à la base du pieu, mais il s'agit d'une plastification encore confinée, qui est due au glissement sous le cisaillement limite sous la pointe.

Sur le plan pratique, l'absence de limitation de la contrainte en pointe ne semble pas présenter d'inconvénient. Par exemple dans le cas d'une fondation placée à la surface d'un sol purement cohérent, la limite théorique du frottement latéral est égale à la cohésion, $q_{5 H}=\mathrm{C}$ et celle de la contrainte en pointe un peu supérieure à cing fois cette valeur. Pour une fondation circulaire, le modèle prévoit alors un début de poinçonnement pour une valeur de la charge $F_{U B}=\sqrt{6} \cdot \pi \cdot C \cdot R^{2}$ alors que la charge de rupture plastique classique serait voisine de $F_{1}=5 . \pi . C . R^{2}$. Il existe donc un rapport de 2,05 environ entre la charge de rupture plastique classique et la charge qui provoque le début de poinçonnement par saturation du cisaillement sous la base. Le rapport correspondant pour les tassements est voisin de 4.

Dans le cas le plus courant, où la fondation est totalement encastrée dans la couche portante, la valeur du rapport passe à environ 4.10 pour les contraintes et à 9 pour les déplacements correspondants. Le poinçonnement par rupture de cisaillement précédera donc toujours largement la rupture par écoulement plastique général.

Ces remarques n'interdisent pas, bien entendu, de vérifier en fin de calcul que la contrainte de pointe reste inférieure à la contrainte limite $q_{\mathrm{B}}$. Mais si la vérification ELU précède le calcul ELS, cette vérification complémentaire est évidemment inutile.

\section{Conclusion}

La question du comportement des fondations profondes sous charge axiale peut sembler entièrement résolue et codifiée. Les discussions qui précèdent montrent que ce n'est pas tout à fait le cas, et qu'il est nécessaire et possible d'imaginer des modèles simples, basés sur des paramètres dont la valeur peut être estimée à partir des essais in situ, qui permettent de reproduire des comportements d'apparence parfois complexe. La réflexion peut être prolongée au-delà.

Il faut remarquer que l'estimation des contraintes limites en pointe est en général basée sur des essais qui correspondent à des ruptures plastiques générales (pénétromètre statique, pression limite pressiométrique). Or, notre modèle prévoit des déformations importantes par plastification confinée bien avant la rupture générale. Et, sur le plan pratique, la rupture d'une fondation profoncle est plus souvent définie par la considération d'un déplacement que par la mesure réelle d'une charge limite.

Il se pourrait donc que la difficulté à faire coïncider les prédictions de rupture à partir des valeurs de capacité limite de pointe avec les résultats d'essai de pieux provienne de la nature différente des deux types de rupture considérés. Dans ces conditions, beaucoup de " capacités de pointe limites » déterminées par des essais de pieux pourraient être des valeurs comprises entre les deux bornes marquées par le premier glissement sous la pointe et la rupture généralisée. Si c'est bien le cas, la résistance de pointe limite permet bien de calculer un ELU, puisque au-delà les déformations ne sont plus limitées. Mais souvent cette limite est atteinte pour des déformations trop importantes pour être mesurables en pratique.

Il n'est pas usuel pour l'instant de réaliser des calculs en déformation sur le comportement des fondations profondes. Les considérations qui précèdent montrent que cela devrait être généralisé, et tout d'abord lors de la réalisation des essais de pieux.

Notre modélisation montre également que la rigidité globale d'une fondation profonde est contrôlée par la rigidité à la base, ce qui confirme l'importance de la qualité du traitement de fond de pieu.

Au total, nous ne maitrisons sans doute pas encore aussi bien que nous l'imaginions le comportement des fondations profondes et il pourrait être fructueux d'améliorer les méthodes de dimensionnement en prenant en considération la question des déformations.

Les résultats qui sont présentés ici sont une partie du travail préliminaire à la réalisation de Profond, un logiciel de calcul du comportement des fondations profondes en cours de mise au point. Ces résultats proviennent de calculs sur tableur, qui ne permettent pas, compte tenu de leur lourdeur, de réaliser toutes les simulations souhaitables qu'il est possible d'attendre d'un logiciel spécialisé. 